\title{
Human Umbilical Cord Mesenchymal Stem-Cell Therapy to Increase the Density of Osteoporotic Mandibular Bone
}

\author{
Nike Hendrijantini ${ }^{1}$ Poedjo Hartono ${ }^{2}$ Muhammad Dimas Aditya Ari ${ }^{1}$ Fedik Abdul Rantan ${ }^{3}$ \\ ${ }^{1}$ Department of Prosthodontic, Faculty of Dental Medicine, \\ Universitas Airlangga, Surabaya, Indonesia \\ 2Department of Obstetrics and Gynecology, Faculty of Medicine, \\ Universitas Airlangga, Surabaya, Indonesia

\begin{abstract}
Address for correspondence Dr. Nike Hendrijantini, DDS, MSc. Specialist in Prosthodontics, Department of Prosthodontic, Faculty of Dental Medicine, Universitas Airlangga, Surabaya, 60132, Indonesia (e-mail: nike-h@fkg.unair.ac.id).
\end{abstract}

${ }^{3}$ Department of Microbiology and Virology, Faculty of Veterinary Medicine, Universitas Airlangga, Surabaya, Indonesia

Eur J Dent 2019;13:58-63

\begin{abstract}
Objective The aim of this study is to evaluate the feasibility of human umbilical cord mesenchymal stem-cell (hUCMSC) therapy in increasing osteoporotic mandibular bone density in a rat model by determining changes in alkaline phosphatase (ALP), osteocalcin, type 1 collagen, and trabecular bone area after treatment.

Materials and Methods This research adopted an experimental posttest-only control group design. Thirty female Wistar rats were randomly divided into six groups, namely, a control group with rats postsham surgery $(\mathrm{T} 1)$, osteoporotic model postovariectomy rats (T2), postovariectomy rats 4 weeks after gelatin injection (T3), postovariectomy rats 8 weeks after gelatin injection (T4), postovariectomy rats 4 weeks after hUCMSC injection (T5), and postovariectomy rats 8 weeks after hUCMSC injection (T6). The rats were all sacrificed for histological and immunohistochemical examinations of ALP, osteocalcin, type 1 collagen, and trabecular bone area.

Results Increased expression of ALP, type 1 collagen, and osteocalcin, as well as increased trabecular bone area, was observed in the treatment groups compared with that in the osteoporotic groups.

Keywords

- human

- mandibular

- osteoporosis

Conclusion hUCMSCs produce significant osteogenic effects and increase osteoporotic mandibular bone density in the animal model. Increases in bone density are demonstrated by the higher levels of ALP, osteocalcin, and type 1 collagen, as well as increases in the trabecular bone area.
\end{abstract}

\section{Introduction}

A dental implant is an optional form of rehabilitation to replace extracted tooth and one required, to a large extent, by elderly patients. ${ }^{1}$ Such individuals, especially females above the age of 50 years, often suffer from menopause-related osteoporosis caused by cessation of ovarian function, which, in turn, decreases estrogen levels in the blood. ${ }^{2}$ Osteoporosis can cause reductions in bone density and quality; ${ }^{3,4}$ in the mandibular bone, a decrease in trabecular density and area can also be observed histologically. These conditions could culminate in dental implant rejection due to the failure of proper osteointegration between the implants and the bone. ${ }^{5}$

Human umbilical cord mesenchymal stem cells (hUCMSCs) have been considered in tissue refinement research as a possible alternative to stem-cell therapy for various diseases and abnormalities. ${ }^{6}$ Previous research has confirmed that the application of hUCMSCs promotes high levels of osteoblast differentiation in an osteoporotic model, a finding that is supported by increased numbers of osteoblasts and expression of transforming growth factor- $\beta 1$ (TGF- $\beta 1$ ) and Runt-related transcription factor 2 (Runx2). ${ }^{7}$ 
After hUCMSCs were shown to produce high levels of osteoblast differentiation, further evaluation was conducted to evaluate their effects on bone formation and mineralization. ${ }^{7}$ As indicators of mandibular bone regeneration, alkaline phosphatase (ALP), osteocalcin, and type 1 collagen expression, as well as the trabecular bone area, can be used to confirm the occurrence of bone formation and mineralization. Increased expression of ALP, osteocalcin, and type 1 collagen indicates the occurrence of osteogenic differentiation. ${ }^{8}$ Osteoblasts undergo mineralization by producing and excreting bone matrix. The main protein product formed in this process is type I collagen, although osteocalcin and several other minor proteins are also present. ${ }^{9,10}$ Osteoblasts express ALP, which promotes mineralization, ${ }^{11}$ through a process that commences in the trabecular bone 30 days after bone resorption and is terminated after 90 days. ${ }^{12}$

Based on the previous studies, research into the potential of hUCMSCs to improve osteoporotic mandibular bone density during bone maturation must be conducted using the increased expression of ALP, osteocalcin, and type 1 collagen, as well as increases in the trabecular bone area, as indicators.

\section{Materials and Methods}

This research was performed to continue the work conducted by Hendrijantini et al, ${ }^{7}$ which aimed to analyze the expression of ALP, type 1 collagen, and osteocalcin and observe the extension of trabecular bone. This research was approved by the ethical health research commission of RSUD, Soetomo Surabaya and the Faculty of Veterinary Medicine, Airlangga University.

Sample preparation and grouping were conducted as in the previous work. ${ }^{7}$ In the present study, paraffin blocks from each of six groups, including a control group with rats postsham surgery (T1), osteoporotic model rats postovariectomy (T2), postovariectomy rats 4 weeks after gelatin injection (T3), postovariectomy rats 8 weeks after gelatin injection (T4), postovariectomy rats 4 weeks after hUCMSC injection (T5), and postovariectomy rats 8 weeks after hUCMSC injection (T6) were immunohistochemically stained.

\section{Staining and Microscopic Examination of Specimens}

Samples were stained using hematoxylin solution (Mayers; Sigma Aldrich, St Louis, Missouri, United States). Immunohistochemical staining using ALP rabbit antihuman monoclonal antibody LS-B6663 (LifeSpan Biosciences Inc.,
Seattle, United States), rabbit anticollagen I polyclonal antibody (BIOSS, United States), and osteocalcin monoclonal antibody mouse IgG1 (Clone \#190125; R\&D Systems, United States) was also conducted. Microscopic observation was performed using a light microscope (Nikon H600 L, Tokyo, Japan) equipped with a DS-Fi2 300-megapixel digital camera and Nikon Image System picture editing software. Data of ALP, type 1 collagen, and osteocalcin expression were calculated using a Remelle for immunohistochemistry index scale from five different fields of view at $\times 400$ magnification. Extension of the trabecular bone area was studied at $\times 200$ magnification.

\section{Statistical Analysis}

Statistical analysis was performed using ANOVA with SPSS software version 15.0 (SPSS, Inc., Chicago, Illinois, United States). $p<0.05$ was considered to indicate statistically significant differences.

\section{Results}

\section{Expression of Alkaline Phosphatase in Rat Mandibular Bone}

Microscopic changes in ALP expression are shown in - Fig. 1. ALP expression in each group is depicted as means and standard deviations in - Fig. 2. An increase in ALP expression was found in groups treated with hUCMSCs compared with that in osteoporosis groups, but no statistically significant difference was found between the groups ( $p=0.245)$.

\section{Expression of Type 1 Collagen in Rat Mandibular Bones} The microscopic expression of osteoblasts is shown clearly by the arrows in -Fig. 3. Type 1 collagen expression in each group is depicted as means and standard deviations in -Fig. 4. A significant increase in type 1 collagen expression was observed in rats treated with hUCMSCs compared with rats in other groups $(p=0.000)$.

\section{Osteocalcin Expression in Rat Mandibular Bone}

The microscopic expression of osteocalcin is clearly shown by the arrows in - Fig. 5. Osteocalcin expression in each group is depicted as means and standard deviations in - Fig. 6. While an increase in osteocalcin expression was observed in groups treated with hUCMSCs compared with that in other groups, the difference observed was not statistically significant $(p=0.219)$.

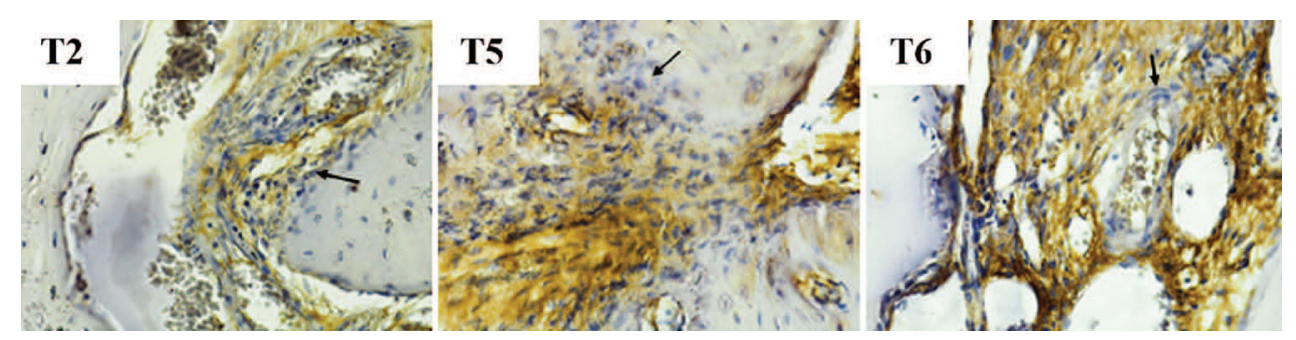

Fig. 1 Results of microscopic examination of alkaline phosphatase expression in the rat mandibles. The arrows show the expression of alkaline phosphatase in the immunoreactive osteogenic cells $(\times 400)$. 


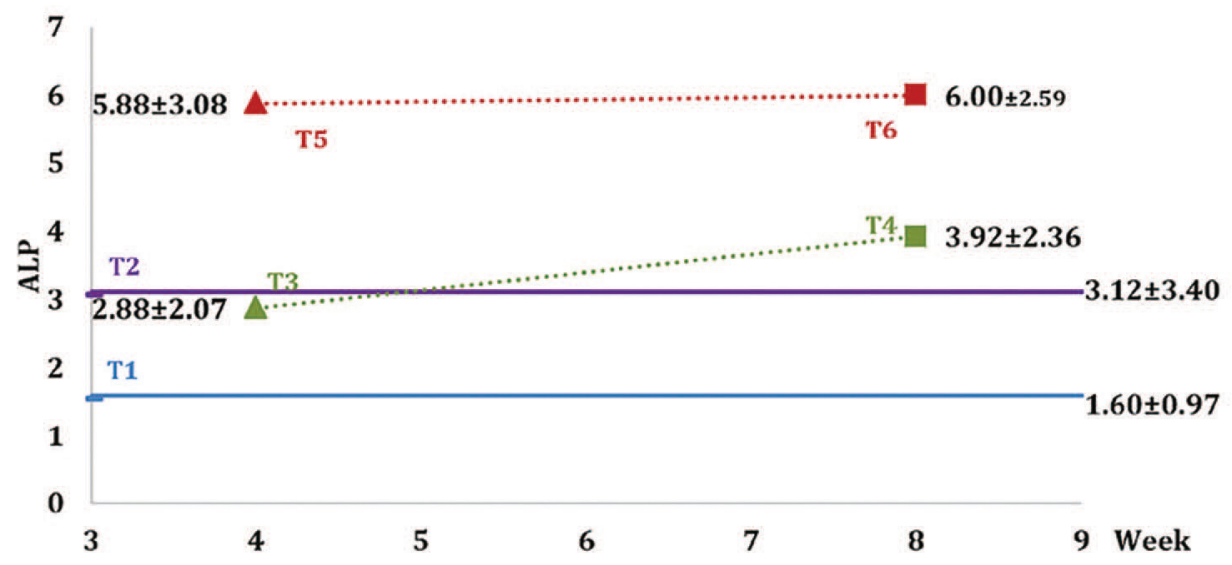

Fig. 2 Graph of mean values and standard deviation of each treatment group for alkaline phosphatase (ALP) expression.

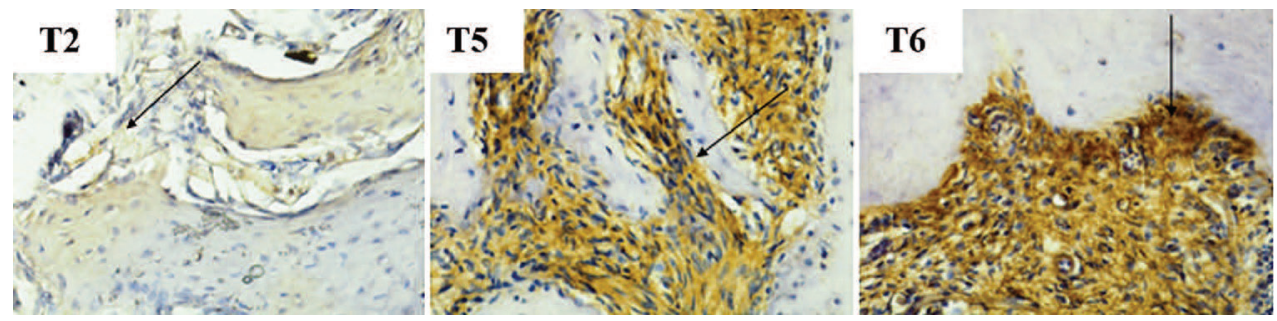

Fig. 3 Microscopic examination results of type 1 collagen expression in the rat jaws. The arrows show the expression of type 1 collagen in the immunoreactive osteogenic cells $(\times 400)$.

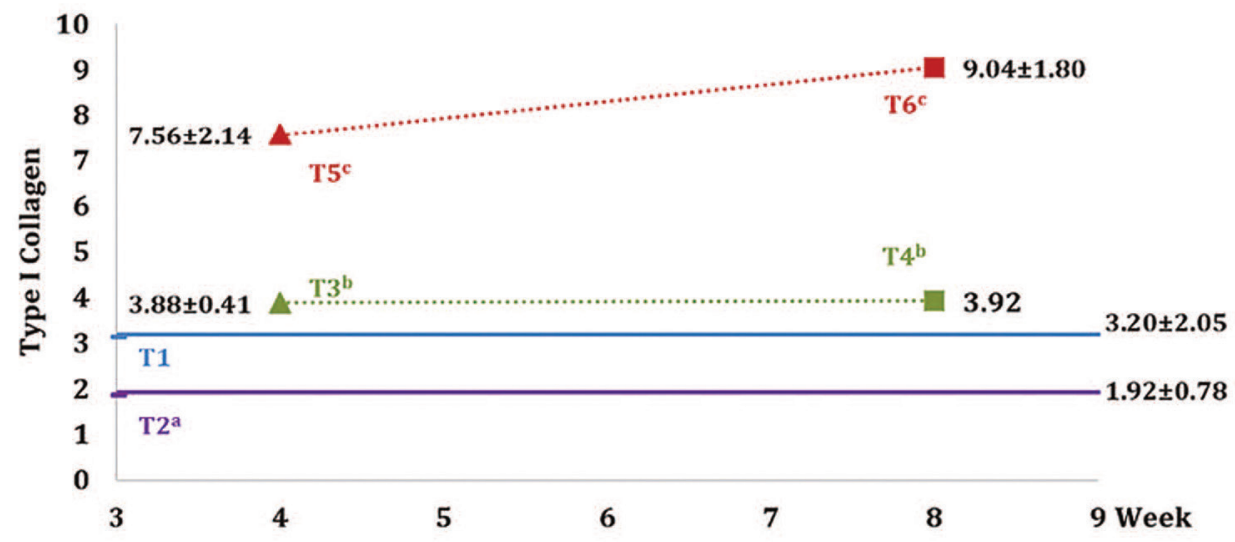

Fig. 4 Graph of mean values and standard deviation of each treatment group in the number of osteoblasts. Different superscripts showed a statistically significant difference $(p<0.05)$ according to the multiple comparison least significant difference least significant difference test.

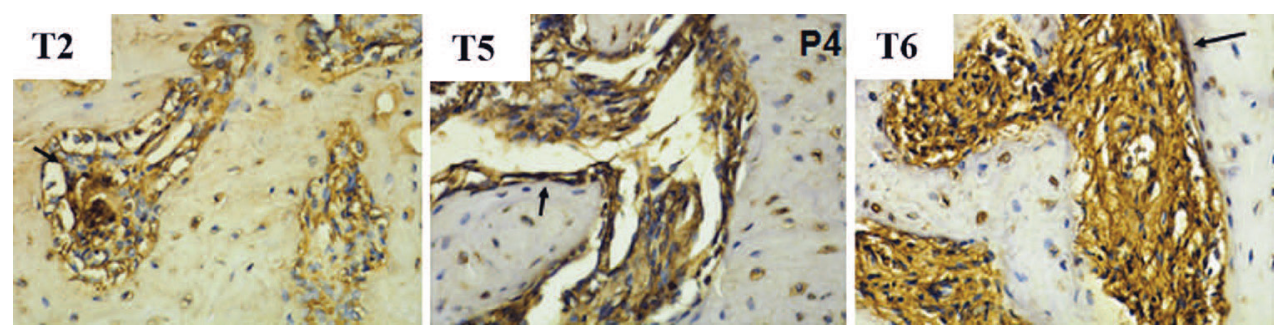

Fig. 5 Results of microscopic examination of osteocalcin expression in the rat jaws. The arrows show the expression of osteocalcin in the immunoreactive osteogenic cells $(\times 400)$. 


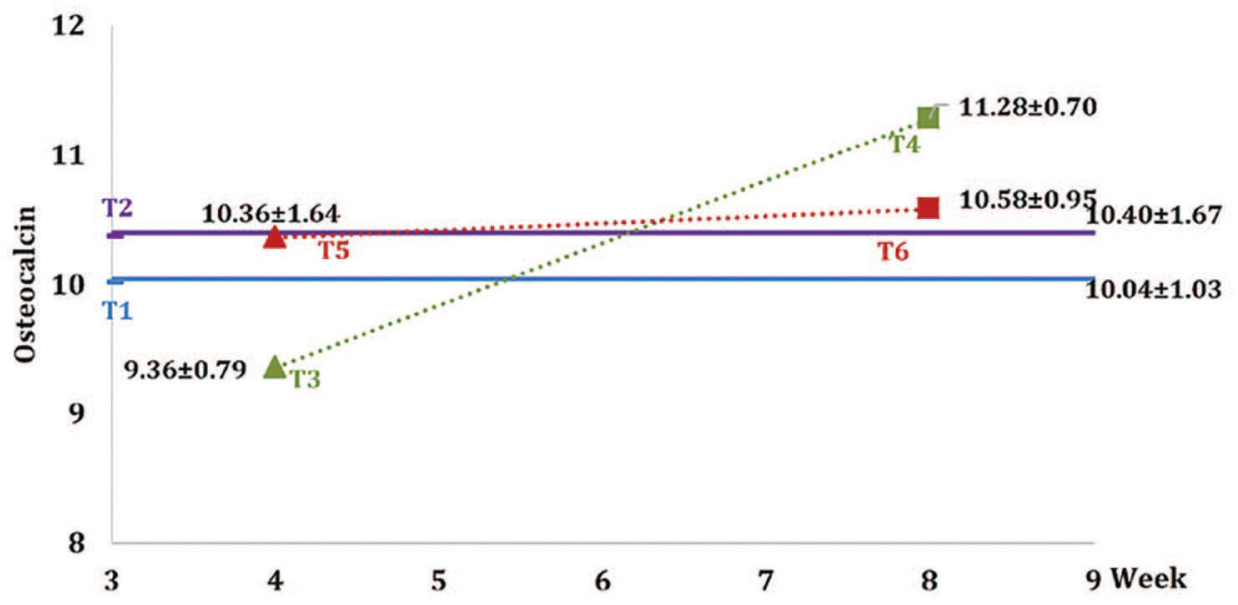

Fig. 6 Graph of mean values and standard deviation of each treatment group in osteocalcin expression.

\section{Extension of the Trabecular Bone Area in Rat Mandibular Bone}

The microscopic area of the trabecular bone is shown clearly by the arrows in - Fig. 7. The extension of the trabecular bone area in each group is depicted as means and standard deviations in -Fig. 8. A significant increase in the trabecular bone area was observed in groups receiving hUCMSCs compared with that of the osteoporosis groups $(p=0.002)$.

\section{Discussion}

The present research confirms that hUCMSCs can promote MSC differentiation in the osteoblast cells. Thus, further evaluation was conducted to understand the ability of these cells to undergo mineralization and bone formation. Here, specific bone formation markers, including ALP, type 1 collagen, and osteocalcin, were examined. Moreover, measurement of the
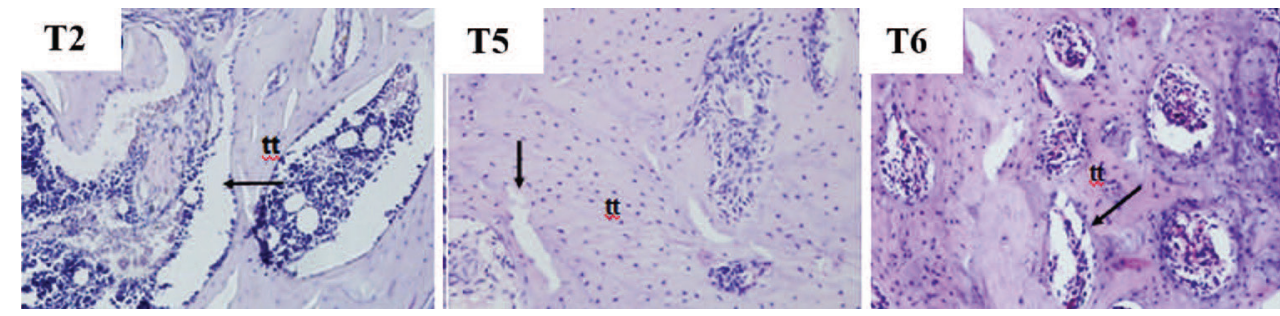

Fig. 7 Large microscopic picture of the trabeculae in the rat jaws. The arrows show the widening of the bone marrow lumen as a result of trabecular bone thinning $(\mathrm{tt})(\times 200)$.

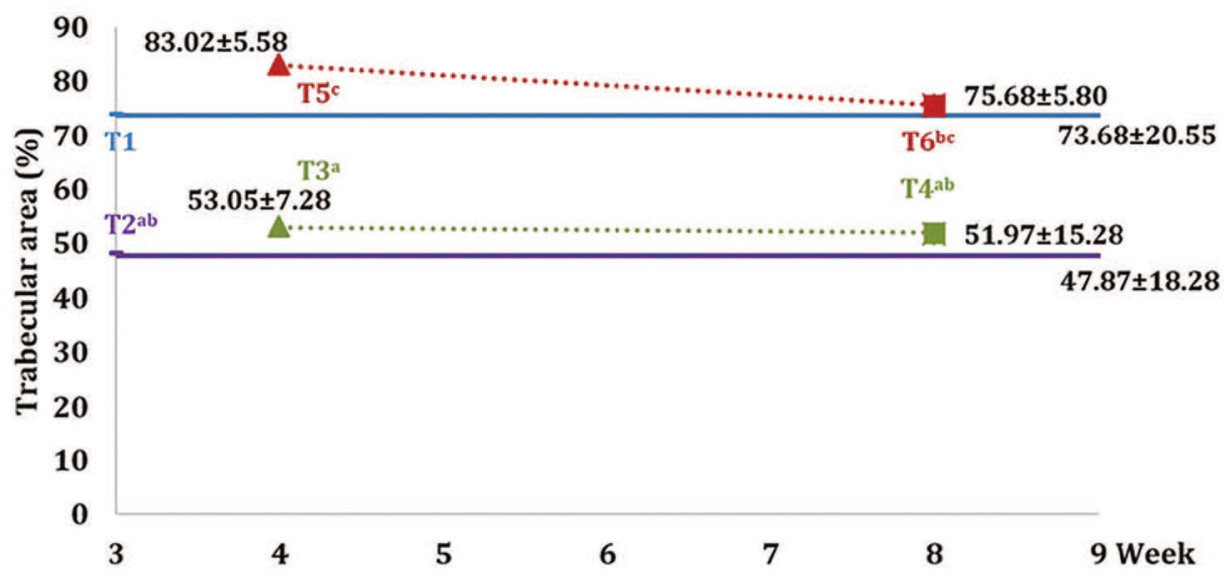

Fig. 8 Graph of mean values and standard deviation of each treatment group on the area of bone trabeculae. Different superscripts showed a statistically significant difference $(p<0.05)$ according to Games-Howell multiple comparison. 
trabecular bone area was performed to obtain an understanding of bone mass increases after hUCMSC treatment.

MSCs in the osteoporotic bones experience a decrease in osteogenic ability, which is marked by the reduced production of TGF- $\beta$, ALP activity, and the production of type 1 collagen in the extracellular matrix. ${ }^{13}$ Prall et al showed that the decreased production of type 1 collagen in the osteoporotic MSCs is due to a reduction in proliferation capability. Decreases in Runx2 in the MSCs also cause stimulus disturbances on some transcription genes in osteoblasts, such as osteocalcin. ${ }^{14}$ Ma et al showed that osteoporotic rat arms treated with MSCs present signs of ossification with the increased expression of Runx2, ALP, and osteocalcin $(p<0.05)$ compared with the control arm. ${ }^{15} \mathrm{Li}$ et al supported these research findings by demonstrating the increased expression of osteocalcin under the same research conditions using the osteoporotic rat models. ${ }^{16}$

MSCs can promote the differentiation of the osteoblasts given a suitable environment and stimuli. No definitive markers exist for the cells of osteogenic lineage, although certain unique contrasting characteristics have been identified. During osteogenic differentiation, MSCs express specific genes, such as ALP, osteopontin, osteocalcin, and type 1 collagen. Increases in the expression of these markers indicate the occurrence of osteogenic differentiation. When the osteoprogenitor develops new bone, ALP activity first decreases and then increases as soon as the bone matrix differentiation and maturation occur. After osteoblasts become osteocytes, ALP activity decreases once more. Osteocalcin is thought to be an end-marker of the mature osteoblasts present in osteocytes, as only a limited number of bones express osteocalcin during early bone formation. ${ }^{17}$ Both phenomena can explain the results of the present research, which revealed the high levels of ALP and osteocalcin despite a lack of significant differences resulting from variations in each step of bone formation.

Type 1 collagen expression was higher in the gelatin group than in other groups because this treatment consists of a protein and peptide mixture derived from collagen; however, gelation does not appear to play a role in the bone formation. Addition of gelatin does not increase bone formation, such as evidenced by Tabata, who showed that treatment with hydrogel gelatin only cannot increase bone mineral density (BMD) significantly. ${ }^{18}$ The present research also showed that type 1 collagen expression is significantly higher in hUCMSC-treated rats than in rats treated with gelatin alone.

Certain anabolic drugs can increase bone density in postovariectomy rats by increasing MSC and mature osteoblast activity. ${ }^{19}$ MSCs were administered directly by local injection and proven to significantly increase the trabecular bone area. Bone formation is dependent on the number and activity of osteoblasts. Increases in osteoblast activity and number can thus increase trabecular bone volume and width while also improving bone microarchitecture repair by creating good trabecular tissue. ${ }^{19}$ Previous research, such as that conducted by Ichioka et al, indicated that the allogenic transplantation of MSCs in rats involving systemic bone marrow mesenchymal stem cells (BMMSC) injection could increase overall trabecular bone density. ${ }^{20}$ Wang et al also conducted research involving the administration of MSCs to postovariectomy rabbits and transplantation of the cells into the distal femur. An increase in trabecular bone width and microstructure due to the development of new osteoids was recorded 8-week postimplantation. ${ }^{21}$ Research by Ma et al, which combined microcomputed tomography and histologic examination, showed that systemic BMMSC application can improve BMD and trabecular bone structure. ${ }^{15}$

In this research, locally transplanted hUCMSCs injected into the mandibular bone were proven to be capable of undergoing in vivo osteogenesis. Larger numbers of active osteoblasts increase mineralization and bone formation, as indicated by the prevalence of ALP, type 1 collagen, and osteocalcin as the main protein in the bones and the wider area of the mandibular trabecular bone.

\section{Conclusion}

Increased expression of ALP, type I collagen, and osteocalcin and a larger trabecular bone area in osteoporotic rats induced by hUCMSCs is the proof of increased osteoblastogenesis. The results of this research confirm the potential success of mandibular bone tissue engineering through external hUCMSC induction.

\section{Financial Support and Sponsorship \\ Nil.}

\section{Conflicts of Interest}

None declared.

\section{Acknowledgments}

The authors gratefully acknowledge the financial support provided by the Rector for Research Funding of Airlangga University, managed by the Community Research and Service Institute, Airlangga University, as an Excellent Research Institution of Higher Education No. 546/Panke.KKE/IX/2017. This research is also supported by the Stem Cell Research and Development Center, Universitas Airlangga.

\section{References}

1 Schimmel M, Müller F, Suter V, Buser D. Implants for elderly patients. Periodontol 2000 2017;73(1):228-240

2 Guyton AC, Hall JE. Text Book of Medical Physiology. 11th ed. Jakarta: EGC Medical Publisher; 2007:1070-6

3 Leslie WD, Tsang JF, Caetano PA, Lix LM; Manitoba Bone Density Program. Effectiveness of bone density measurement for predicting osteoporotic fractures in clinical practice. J Clin Endocrinol Metab 2007;92(1):77-81

4 Kling JM, Clarke BL, Sandhu NP. Osteoporosis prevention, screening, and treatment: a review. J Womens Health (Larchmt) 2014;23(7):563-572

5 Misch CE. Rationale for dental implants. In: Contemporary Implant Dentistry. 3rd ed. St. Louis, Canada: Mosby Inc.; 2008:3-21

6 Romanov YA, Svintsitskaya VA, Smirnov VN. Searching for alternative sources of postnatal human mesenchymal stem cells: candidate MSC-like cells from umbilical cord. Stem Cells 2003;21(1):105-110 
7 Hendrijantini N, Kusumaningsih T, Rostiny R, Mulawardhana P, Danudiningrat CP, Rantam FA. A potential therapy of human umbilical cord mesenchymal stem cells for bone regeneration on osteoporotic mandibular bone. Eur J Dent 2018;12(3):358-362

8 Rutkovskiy A, Stensløkken KO, Vaage IJ. Osteoblast differentiation at a glance. Med Sci Monit Basic Res 2016;22:95-106

9 Florencio-Silva R, Sasso GR, Sasso-Cerri E, Simões MJ, Cerri PS. Biology of bone tissue: structure, function, and factors that influence bone cells. BioMed Res Int 2015;2015:421746

10 Blair HC, Larrouture QC, Li Y, et al. Osteoblast differentiation and bone matrix formation in vivo and in vitro. Tissue Eng Part B Rev 2017;23(3):268-280

11 Orimo $\mathrm{H}$. The mechanism of mineralization and the role of alkaline phosphatase in health and disease. J Nippon Med Sch 2010;77(1):4-12

12 Kini U, Nandeesh BN. Physiology of bone formation, remodeling, and metabolism. In: Fogelman I, Gnanasegaran G, Van der Wall $\mathrm{H}$, eds. Radionuclide and Hybrid Bone Imaging. Berlin: Springer; 2012:29-57

13 Pino AM, Rosen CJ, Rodríguez JP. In osteoporosis, differentiation of mesenchymal stem cells (MSCs) improves bone marrow adipogenesis. Biol Res 2012;45(3):279-287

14 Prall WC, Haasters F, Heggebö J, et al. Mesenchymal stem cells from osteoporotic patients feature impaired signal transduction but sustained osteoinduction in response to BMP-2 stimulation. Biochem Biophys Res Commun 2013;440(4):617-622
15 Ma L, Aijima R, Hoshino Y, et al. Transplantation of mesenchymal stem cells ameliorates secondary osteoporosis through interleukin-17-impaired functions of recipient bone marrow mesenchymal stem cells in MRL/lpr mice. Stem Cell Res Ther 2015;6:104

16 Li C, Wei G, Gu Q, Wang Q, Tao S, Xu L. Proliferation and differentiation of rat osteoporosis mesenchymal stem cells (MSCs) after telomerase reverse transcriptase (TERT) transfection. Med Sci Monit 2015;21:845-854

17 Kaveh K, Ibrahim R, Bakar M, Ibrahim T. Mesenchymal stem cells, osteogenic lineage and bone tissue engineering: a review. J Anim Vet Adv 2011;10:2317-2330

18 Tabata Y. Tissue regeneration based on drug delivery technology. In: Ashammakhi N, Ferretti P, eds. Topics in Tissue Engineering. Vol. 1. Tamahe, Finlandia: University of Oulu; 2003:1-32

19 Marie PJ, Kassem M. Osteoblasts in osteoporosis: past, emerging, and future anabolic targets. Eur J Endocrinol 2011;165(1):1-10

20 Ichioka N, Inaba M, Kushida $\mathrm{T}$, et al. Prevention of senile osteoporosis in SAMP6 mice by intrabone marrow injection of allogeneic bone marrow cells. Stem Cells 2002;20(6):542-551

21 Wang Z, Goh J, Das De S, et al. Efficacy of bone marrow-derived stem cells in strengthening osteoporotic bone in a rabbit model. Tissue Eng 2006;12(7):1753-1761 